\title{
Controlling Two-Photon Action Cross Section by Changing a Single Heteroatom Position in Fluorescent Dyes
}

\author{
Borys Ośmiałowski,* Elizaveta F. Petrusevich, Magda A. Antoniak, Izabela Grela, \\ Mohammed A. Bin Jassar, Marcin Nyk, Josep M. Luis, Beata Jędrzejewska, Robert Zalésny,* \\ and Denis Jacquemin*
}

Cite This: J. Phys. Chem. Lett. 2020, 11, 5920-5925

Read Online

\begin{abstract}
ACCESS
Wll Metrics \& More

Article Recommendations

ABSTRACT: The optimization of nonlinear optical properties for "real-life" applications remains a key challenge for both experimental and theoretical approaches. In particular, for two-photon processes, maximizing the two-photon action cross section (TPACS), the figure of merit for two-photon bioimaging spectroscopy, requires simultaneously controlling all its components. In the present Letter, a series of difluoroborates presenting various heterocyclic rings as an electron acceptor have been synthesized and their absorption, fluorescence, photoisomerization, and two-photon absorption features have been analyzed using both experimental and theoretical approaches. Our results demonstrate that the TPACS values can be fine-tuned by changing the position of a single heteroatom, which alters the fluorescence quantum yields without changing the intrinsic two-photon absorption cross section. This approach offers a new strategy for optimizing TPACS.
\end{abstract}

Supporting Information

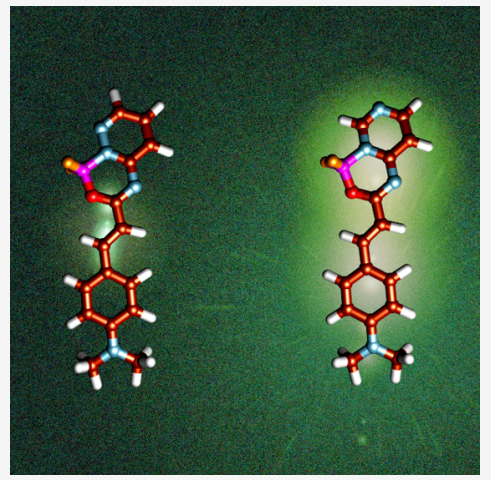

$\mathrm{T}$ wo-photon absorption (2PA), a phenomenon predicted nearly a century ago, ${ }^{1}$ has a huge potential in several fields, e.g., in microfabrication, photodynamic therapy, threedimensional data storage, and bioimaging. ${ }^{2-5}$ The interest of $2 \mathrm{PA}$ is also illustrated by the rapid development of two-photon microscopy. For that purposes two-photon probes should fulfill several requirements: (i) have large two-photon action cross section (TPACS), which is the product of two-photon absorption cross section $\left(\sigma^{2 \mathrm{PA}}\right)$ and fluorescence quantum yield $(\Phi)$, exceeding $50 \mathrm{GM}^{6}$ (ii) bind selectively to a target; and (iii) be photostable. Moreover, as the two-photon excited fluorescence applied in bioimaging ${ }^{7}$ typically operates in the $650-1100 \mathrm{~nm}$ optical window, ${ }^{8,9}$ it is necessary to set the onephoton absorption within the $330-550 \mathrm{~nm}$ domain. The common strategy to maximize the $\Phi \times \sigma^{2 \mathrm{PA}}$ product is to enhance $\sigma^{2 \mathrm{PA}}$ typically through an optimal choice of electrondonating and electron-accepting moieties or an extension of the $\pi$-conjugated linker in a push-pull compounds aiming to increase the charge transfer (CT). In the present Letter, we aim at maximizing $\Phi \times \sigma^{2 \mathrm{PA}}$, while preserving the topology of a probe, through fine-tuning of the fluorescence quantum yield in a series of compounds presenting a difluoroborate $\left(\mathrm{BF}_{2}\right)$ group. In fact, we do change only one atom at a time in these dyes. Our interest in this class of compounds stems from their excellent photophysical properties. ${ }^{10-14}$ In general, fluoroborates exhibit an intense and sharp emission with high quantum yields, nanosecond lifetimes, and narrow absorption bands in the visible spectral region characterized by high extinction coefficients. ${ }^{10-12,15}$ In addition, they show a good stability in biological environments-a feature that places them in a privileged spot as compared to many other molecular probe candidates. Moreover, the successful functionalization of BFcarrying compounds to optimize their photochemical properties has been regularly achieved, through substitution, extension of the conjugated path with either double or triple carbon-carbon bonds, planarization, benzoannulation, substitution of the fluorine atoms, replacement of an atom binding Lewis acid, etc. ${ }^{16,17}$ Recently, Yamaji et al. demonstrated that the fluorescence quantum yield in two diazine-based difluoroborates significantly differ from that of the corresponding pyridine derivative, ${ }^{18,19}$ and we decided to adopt a similar strategy in our research to enhance fluorescence quantum yield, thus maximizing TPACS.

In order to assess the effect of the heteroatom position in diazine-carrying donor-acceptor dyes on the TPACS values, we focus in this Letter on the compounds shown in Scheme 1, which also indicates atom numbering, the synthetic path, and the trans and cis forms. Previously, we have shown that $\mathrm{BF}_{2}-$ carrying molecules based on amides exhibit significant twophoton absorption $^{20,21}$ and that the most effective were

Received: May 11, 2020

Accepted: July 6, 2020

Published: July 6, 2020 
Scheme 1. Structures of Studied Compounds in Their Trans and $\mathrm{Cis}$ Conformations

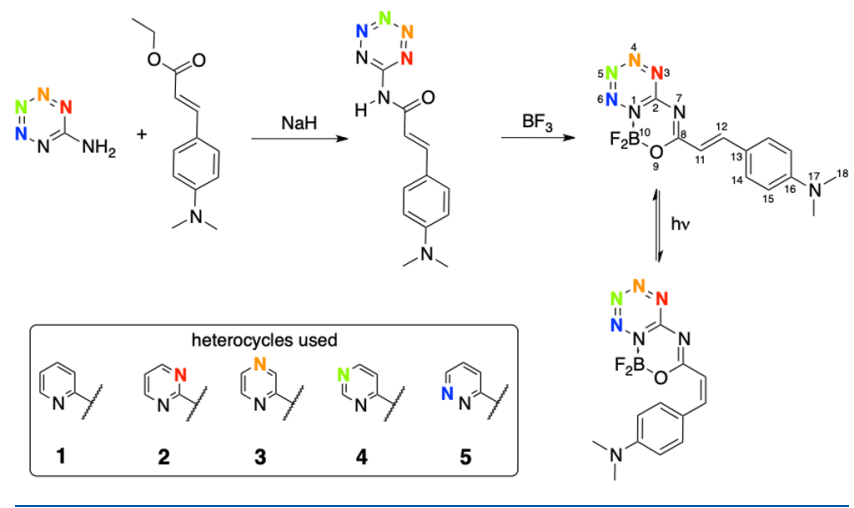

molecules with strong CT character. We stress that only one (or zero as in 1) colored nitrogen atom is present in a given molecule, so the changes are indeed minimal from one dye to another. While the elongated $\pi$-conjugation path between the electron-donating and electron-accepting moieties is beneficial for maximizing two-photon absorption cross section, the presence of an alkenyl moiety may lead to trans-cis photoisomerization. ${ }^{22-28}$ Although the photoisomerization might be desired in photochromic applications, it competes with fluorescence and is thus detrimental for our purposes. That is why this reaction should be controlled, which can be done to some extent, through the introduction of adequate substituents. ${ }^{29,30}$ In one of our previous works, we have shown that the geometry of the electron-donating group determines its donating character and the photoisomerization rate constant, ${ }^{29}$ yet it remains unknown (i) if the photoisomerization is dependent on such subtle structural changes as the ones studied herein and (ii) if changing the position of one nitrogen atom only allows the tuning of the fluorescence quantum yields. For the former aspect, let us mention that the photoisomerization of the double $\mathrm{N}=\mathrm{N}$ bond in phenylazadiazines might indeed be influenced by the position of the nitrogen atom. ${ }^{31}$ Nevertheless, we note that the heterocyclic part of the present dyes is well separated from the carboncarbon double bond and no steric impact on the photoisomerization can be expected. To our knowledge, there is only one previous publication addressing the influence of the nitrogen atom position in isoelectronic $\mathrm{BF}_{2}$ derivatives on properties of dyes. ${ }^{32}$ However, that contribution considered three (out of five) nitrogen positions and, more importantly, those molecules were not photoisomerizable.

The aim of the current Letter is to introduce a new strategy for maximizing the TPACS values solely by changing heteroatom position in heterocyclic ring. To achieve this goal, we (i) study the charge-transfer properties of difluoroborates carrying pyridine and four diazine rings as electron acceptor, (ii) correlate the nitrogen position with twophoton absorption cross sections and fluorescence quantum yields, and (iii) analyze the impact of the putative photoisomerization of the $\mathrm{C}=\mathrm{C}$ moiety on the photophysical responses.

The synthesis (Scheme 1) of the dyes was performed by reaction of the amino-substituted heterocycle with $\mathrm{NaH}$ and next with $(N, N$-dimethylamino) cinnamic ester, obtained as described elsewhere, ${ }^{33}$ in THF solution and, after amide purification (recrystallization from ethanol), the $\mathrm{BF}_{3}$ etherate was used as described earlier ${ }^{34}$ to obtain $\mathbf{1 - 5}$. Note that the spectroscopic properties of $\mathbf{1}$ have been reported by some of us previously. ${ }^{20,34}$ In order to characterize the CT properties of these dyes, the absorption and fluorescence spectra were recorded in a number of solvents of various polarities (see Table S1 in the Supporting Information file). The extinction coefficients of $1-5$ are ca. 2 times higher than that of the parent ethyl 4-(N,N-dimethylamino) cinnamate. ${ }^{35}$ The position of the additional nitrogen in the heterocyclic ring clearly influences the spectroscopic properties; while the fluorescence quantum yield is increasing with the decreasing solvent polarity in $\mathbf{2 - 5}$, the behavior differs significantly in $\mathbf{1}$ (see Figure S70). Indeed, for that dye, the highest $\Phi$ is recorded in THF (0.312) and this value is higher than the $\Phi$ in any solvent of 2,3 , and 5 (the highest $\Phi$ in the whole series is observed for $\mathbf{4}$ in ethers and chloroform). To shed more light onto the possible source of differences in fluorescence quantum yields, we performed the calculations of the two lowest singlet- and triplet-state energies (see Table S5 in the Supporting Information file). It turns out that the $T_{1}$ state of $\mathbf{2 - 5}$ is always below the $S_{1}$ roughly by $0.5 \mathrm{eV}$, whereas the $\mathrm{T}_{2}$ state is higher than $S_{1}$. Hence, judging solely by these energy levels, $S_{1} \rightarrow T_{1}$ intersystem crossing might be a plausible scenario. Consequently, we performed spectroscopic measurements for compounds $\mathbf{2}-\mathbf{5}$ in glassy matrix of 2 -methyltetrahydrofuran (MeTHF). It follows from Figure S71 in the Supporting Information, which presents normalized fluorescence and phosphorescence spectra at $77 \mathrm{~K}$, that compounds 2 and 4 show much larger fluorescence intensity than 3 and 5 in MeTHF, in line with the measurements in $\mathrm{CHCl}_{3}$ solutions (see Figure $\mathrm{S} 70$ and Table 1). Conversely, much larger

Table 1. Fluorescence Quantum Yields ( $\Phi)$, Two-Photon Absorption Cross Sections $\left(\sigma^{2 \mathrm{PA}}\right)$ and TPACS Values for All Studied Compounds in Chloroform Solution ${ }^{a}$

$\begin{array}{cccc}\text { compound } & \Phi & \sigma^{2 \mathrm{PA}}(\mathrm{GM}) & \text { TPACS }(\mathrm{GM}) \\ \mathbf{2} & 0.20 & 404 & 81 \\ 3 & 0.02 & 380 & 8 \\ 4 & 0.46 & 367 & 169 \\ 5 & 0.02 & 280 & 6\end{array}$

${ }^{a}$ Experimental two-photon absorption cross section values correspond to band maxima at $925 \mathrm{~nm}$.

phosphorescence intensity are observed for compounds 3 and 5, hinting that that intersystem crossing is a plausible mechanism behind low FQYs for these two compounds. Moreover, we performed excited-state geometry optimizations of 2,3 , and 4 in chloroform solution, taking into account four explicit solvent molecules. The results of the analysis is presented in the Supporting Information and it shows that 3 has significantly more distorted geometry in the $S_{1}$ excited state, in comparison to 2 and 4 (see Figures S86-S87). The measured Stokes shift clearly hints at a significant CT, the largest Stokes shifts being obtained in $\operatorname{ACN}(1,3$, and 4), acetone (2), and THF (5). Such CT should lead to a quinoidlike geometry in the excited state (Figure S85 in the Supporting Information) changing the character of the central carbon-carbon bond (double bond in the ground state) and also influencing the barrier to rotation around that bond. To shed a complementary light onto the experimental data, we have determined the electron density differences corresponding to the absorption of a photon. A representation for $\mathbf{1 - 5}$ is displayed in Figure S84. Obviously, there is a CT taking place 
from amino group to the core of the fluoroborate dye that respectively act as donor and acceptor groups. According to Le Bahers model, ${ }^{36}$ this CT corresponds to a transfer of 0.67 (0.75) electron over 4.1 (3.6) $\AA$ for the trans (cis) isomer, which indicate a large CT. Similar parameters are obtained for all dyes, there is just a small enhancement of the CT in $\mathbf{2 - 5}$ as compared to 1 due to the stronger accepting properties of the diazine.

Table S2 and Figures S21-S69 in the Supporting Information collects the data related to time-resolved fluorescence measurements. For most of the compound/ solvent pairs, a double exponential fluorescence decay is observed. Several origins can explain the presence of two fluorescent species in solution. For instance, in many CT molecules two forms, differing by the geometry of the donor moiety (twisted/planar intramolecular charge transfer (TICT/ PICT) mechanisms), ${ }^{37}$ might be present. However, this aspect is not the focus of the present Letter, and it is not discussed in the following. The key observation for our purposes is that the spectral data indicate that the brightest compound in the series is 4. Indeed, its attenuation coefficient multiplied by fluorescence quantum yield reaches ca. 26000 in dioxane and chloroform, 20000-21000 in ethyl acetate and THF, while the next brightest dye, 1, displays notably smaller responses, e.g., 15500 in ethyl acetate and 14300 in THF.

Figure 1 shows the initial and final normalized spectra for $\mathbf{1 - 5}$ in acetonitrile solution when irradiated by a laser $(24500$

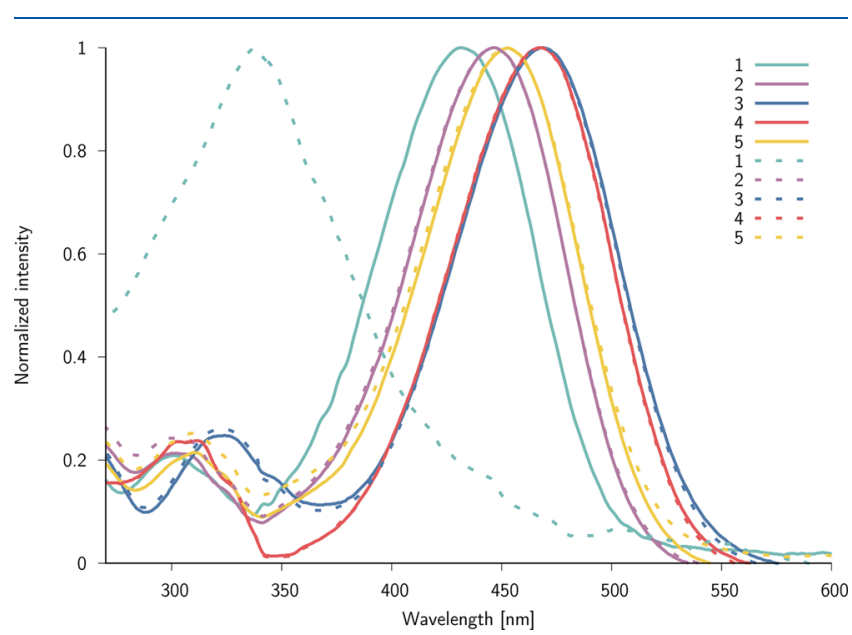

Figure 1. Normalized absorption spectra of compounds in acetonitrile solution (solid lines, initial step) and spectra recorded after irradiation (dashed lines, after ca. $7 \mathrm{~h}$ of irradiation).

$\mathrm{s}, 50 \mathrm{~mW}, 457 \mathrm{~nm})$. The full set of spectra can be found in Figures S72-S76. It is evident that only the spectrum of compound 1 changes upon irradiation looking at the intensity changes at 431 and $337 \mathrm{~nm}$. Such change can originate either in the photoisomerization of the central double bond, or in the decomposition of $\mathbf{1}$ into another species. In contrast, the lack of changes in the spectra of $\mathbf{2 - 5}$ derivatives could be explained by either (i) photoisomerization does not take place or (ii) photoisomerization takes place but the cis isomer has a very similar absorption maximum (and attenuation coefficient) as its trans counterpart. However, taking into account the differences between initial and irradiated responses for 1, (ii) is not probable, a fact also confirmed by simulations of oneand two-photon absorption spectra (see the Supporting Information, Figures S88-S91). Moreover, the absorption of the irradiated solution increased (by ca. $2 \%$ ) in the $400-440$ $\mathrm{nm}$ region and decreased in the 320-360 $\mathrm{nm}$ domain after storing the solution in a closed cuvete at room temperature overnight. Therefore, the absorption studies of the irradiated solutions indicate that neither the photoisomerization nor decomposition takes place for $\mathbf{2 - 5}$, in contrast to $\mathbf{1}$, which undergoes photoswitching. We note that the photoisomerization of $\mathbf{1}$ is not effective compared to cationic dyes having a similar skeleton, that reached the photostationary state in $500 \mathrm{~s}$ of irradiation, ${ }^{38}$ whereas $24500 \mathrm{~s}$ were needed for $\mathbf{1}$ to almost complete the isomerization.

To assess the potential for bioimaging applications, we have studied the two-photon absorption spectra of compounds that do not exhibit detrimental photoisomerization (2-5). To that end we employed both experimental and theoretical approaches (see the Supporting Information for details). The experimental two-photon absorption spectra measured in a wide spectral range using the Z-scan technique is shown in Figure 2. The two-photon spectra of all compounds share

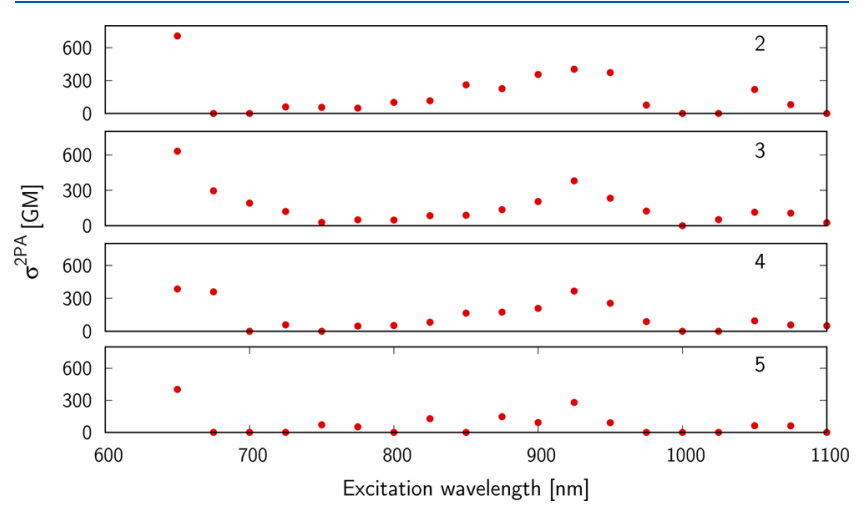

Figure 2. Experimental two-photon absorption spectra of studied compounds.

several common features with (i) a band with maximum at ca. $925 \mathrm{~nm}$ (corresponding to the $S_{0} \rightarrow S_{1}$ excitation, see Figure $1)$, (ii) a peak of two-photon absorption cross section in the 280-404 GM range, and (iii) even higher two-photon transition responses for higher-lying states (in the 600-700 $\mathrm{nm}$ range). Therefore, the modification of the position of the nitrogen atom has apparently a trifling impact on the twophoton absorption intensity corresponding to the longwavelength $\pi \rightarrow \pi^{*}$ transition. Obviously, the two-photon excitations of 2-5 correspond to excitation of the more stable trans conformer, whereas in $\mathbf{1}$ there could be (undesired) contributions from cis forms, as the two-photon excitation might induce trans-cis photoisomerization. ${ }^{39}$ In order to shed light on this interesting aspect, i.e., how different might be the two-photon responses of the two isomers of $\mathbf{1 - 5}$, we performed state-of-the-art electronic structure calculations using a $\mathrm{QM} / \mathrm{MM}$ approach. More precisely, for trans and cis conformers of compounds 1-5, we performed second-order coupled-cluster (CC2) calculations using electrostatic embedding approach to represent discrete solvent environment. The results of statistical analysis are shown in Table S6 in the Supporting Information. First, one notes that the experimental ordering of the maxima $\left[\lambda_{\max }(1)<\lambda_{\max }(2) \approx \lambda_{\max }(5)<\lambda_{\max }\right.$ $\left.(4) \approx \lambda_{\max }(3)\right]$ is nicely reproduced by these calculations. A blue shift of the theoretical excitation wavelengths with respect to experimental data is likely due to the lack of vibronic couplings in the calculations and the good but not perfect 
accuracy of the employed electronic-structure method (CC2). Second, for all compounds the new band corresponding to the $S_{0} \rightarrow S_{1}$ transition for cis isomers is red-shifted as compared to the $S_{0} \rightarrow S_{1}$ band for trans isomers, which is in agreement with available experimental data for 1 . The simulated two-photon absorption spectra for all compounds (trans and cis forms) are shown in Figures S90 and S91. Two key observations can be drawn from their analysis: (i) all trans isomers exhibit similar values of two-photon $S_{0} \rightarrow S_{1}$ transition strengths, which is in excellent agreement with experimental data; (ii) the cis isomers show slightly lower values of two-photon $S_{0} \rightarrow S_{1}$ transition strengths in comparison to their trans counterparts. Based on the analysis presented above, we can now analyze the TPACS values presented in Table 1 . As it is seen, TPACS vary to a large extent from 6 (compound 5) up to 169 GM (compound 4). As already discussed, the two-photon absorption cross section values remain in the 280-404 GM range, so the huge changes in TPACS values can be largely attributed to differences in fluorescence quantum yields.

In order to analyze the two-photon activity, we have employed the generalized few-state model (GFSM) ${ }^{40}$ recently developed for electronic structure theories with a nonhermitian structure. $^{41}$ GFSM allows to interpret the two-photon transition strengths in terms of electronic structure parameters:

$$
\left\langle\delta_{0 J}^{\mathrm{GFSM}}\right\rangle=\sum_{K} \sum_{L} \delta_{0 J K L}^{\mathrm{GFSM}}
$$

where

$$
\begin{aligned}
& \delta_{0 J K L}^{\mathrm{GFSM}}=\frac{2}{15 \Delta E_{K} \Delta E_{L}}(\alpha+\beta) \\
& \alpha=\left|\mu^{J K}\left\|\mu^{K 0}\right\| \mu^{0 L} \| \mu^{L J}\right| \times \\
& \left(\cos \theta_{J K}^{K 0} \cos \theta_{0 L}^{L J}+\cos \theta_{J K}^{0 L} \cos \theta_{K 0}^{L J}+\cos \theta_{J K}^{L J} \cos \theta_{K 0}^{0 L}\right) \\
& \beta=\left|\mu^{J L}\left\|\mu^{L 0}\right\| \mu^{0 K} \| \mu^{K J}\right| \times \\
& \left(\cos \theta_{J L}^{L 0} \cos \theta_{0 K}^{K J}+\cos \theta_{J L}^{0 K} \cos \theta_{L 0}^{K J}+\cos \theta_{J L}^{K J} \cos \theta_{L 0}^{0 K}\right)
\end{aligned}
$$

In eq 1 , the superscripts distinguish between the right $(L 0)$ and the left $(0 L)$ moments and $\Delta E_{K}=\frac{1}{2} \omega_{J}-\omega_{K}$. The $\theta_{P Q}^{R S}$ terms represents the angle between the transition dipole moment vectors $\mu^{P Q}$ and $\mu^{R S}$. Although the summations in eq 1 for $K$ and $L$ run over all the electronic states, any number of intermediate states $K$ and $L$ can be chosen to obtain an approximated value. Here we use both two- and three-state models (3SM, $S_{2}$ taken as intermediate state), in which $K$ and $L$ can be either the ground state 0 , the final excited state $J$ (two-state model), or an intermediate state (three-statemodel). The comparison of the two models is shown in Table S7 and allows us to conclude that the two-state model is adequate to analyze two-photon transition strengths, as it accurately restores the ordering of the two-photon transition strengths in the studied series of compounds. Figure 3 presents the individual terms contributing to average two-photon transition strength, showing that the property in question (being proportional to the experimentally measured twophoton absorption cross sections) undergoes much smaller variations than individual terms related to electronic structure parameters. In other words, the moderate changes in twophoton absorption cross sections across the series $\mathbf{2 - 5}$ are due to cancellations of competing terms. The largest variations are observed in the $\delta_{0111}$ term, which encompasses the product of

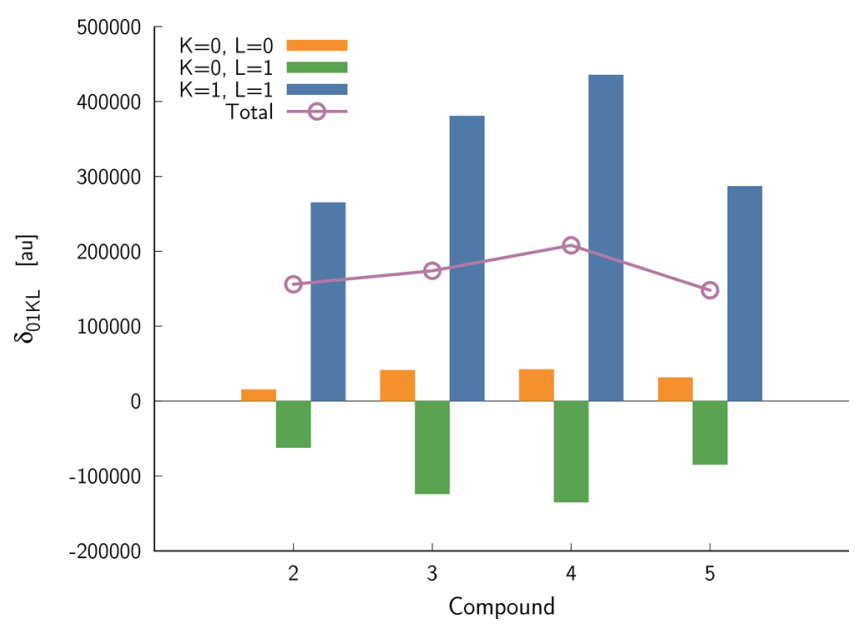

Figure 3. Decomposition of the two-photon transition strength based on the generalized two-state model. All calculations were performed at the CC2 level for molecules in $\mathrm{CHCl}_{3}$ solution. Note that the term corresponding to $K=1, L=0$ has an equal value as that labeled by $K$ $=0, L=1$ and is not shown for the sake of clarity.

the square of $S_{0} \rightarrow S_{1}$ transition moment, $\left|\mu^{01}\right|^{2}$ and the square of the dipole moment in the $S_{1}$ electronic excited state, $\left|\mu^{11}\right|^{2}$. The variations of the $\delta_{0111}$ term correlate well with the changes in the excited-state dipole moment when going from 2 to 5 (see Table S4). We note that both the experimental (LippertMataga correlations, see Table S4 and Figures S77-S81) and theoretical (Table S4) data show large dipole moment changes upon excitation (exceeding $10 \mathrm{D}$ ), which is heteroatomplacement specific.

In short, we have shown in this Letter that one can maximize the TPACS value, the figure of merit for bioimaging applications, by keeping the two-photon absorption crosssection (almost) constant but effectively controlling the emission quantum yield. Such optimization does not necessarily require using longer linkers nor choosing stronger donor/acceptor groups, since changing the position of a single heteroatom might be sufficient. We note that three of the molecules discussed here have TPACS values larger than 50 GM and are thus good candidates for bioimaging applications, ${ }^{6}$ illustrating the potential of this new approach.

\section{ASSOCIATED CONTENT}

\section{St Supporting Information}

The Supporting Information is available free of charge at https://pubs.acs.org/doi/10.1021/acs.jpclett.0c01438.

Description of synthetic procedure, characterization of compunds, NMR spectra, absorption and fluorescence measurements, tables of spectral properties, timeresolved fluorescence spectra, fluorescence quantum yields, phosphorescence and fluorescence spectra, Stokes shift vs $f(\varepsilon, n)$ and vs $\Delta f_{\mathrm{LM}}, \mathrm{Z}$-scan setup and experiment and Z-scan traces, results of $\mathrm{QM} / \mathrm{MM}$ calculations, electron density difference maps, charge transfer scheme, excited-state geometries, electronic one- and two-photon absorption spectra, and tables of excitation energies, excitation wavelengths, and transition strengths (PDF) 


\section{AUTHOR INFORMATION}

\section{Corresponding Authors}

Borys Ośmiałowski - Faculty of Chemistry, Nicolaus Copernicus University, PL-87100 Torun, Poland; (1) orcid.org/ 0000-0001-9118-9264; Email: Borys.Osmialowski@umk.pl

Robert Zaleśny - Department of Physical and Quantum Chemistry, Faculty of Chemistry, Wroctaw University of Science and Technology, PL-50370 Wroctaw, Poland; (1) orcid.org/ 0000-0001-8998-3725; Email: Robert.Zalesny@pwr.edu.pl

Denis Jacquemin - Universite de Nantes, CNRS, CEISAM UMR 6230, F-44000 Nantes, France; 10 orcid.org/00000002-4217-0708; Email: Denis.Jacquemin@univ-nantes.fr

\section{Authors}

Elizaveta F. Petrusevich - Department of Physical and Quantum Chemistry, Faculty of Chemistry, Wroctaw University of Science and Technology, PL-50370 Wroctaw, Poland

Magda A. Antoniak - Advanced Materials Engineering and Modelling Group, Faculty of Chemistry, Wroctaw University of Science and Technology, PL-50370 Wroctaw, Poland; (1) orcid.org/0000-0003-1043-6089

Izabela Grela - Faculty of Chemical Technology and Engineering, UTP University of Science and Technology, 85-326 Bydgoszcz, Poland

Mohammed A. Bin Jassar - Université d'Aix-Marseille, Département de Chimie, CNE Master, 13013 Marseille, France

Marcin Nyk - Advanced Materials Engineering and Modelling Group, Faculty of Chemistry, Wroctaw University of Science and Technology, PL-50370 Wroctaw, Poland; (1) orcid.org/00000002-0329-4038

Josep M. Luis - Institute of Computational Chemistry and Catalysis and Department of Chemistry, University of Girona,

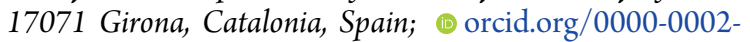
2880-8680

Beata Jędrzejewska - Faculty of Chemical Technology and Engineering, UTP University of Science and Technology, 85-326 Bydgoszcz, Poland; (1) orcid.org/0000-0002-3406-5828

Complete contact information is available at:

https://pubs.acs.org/10.1021/acs.jpclett.0c01438

\section{Notes}

The authors declare no competing financial interest.

\section{ACKNOWLEDGMENTS}

B.O., M.N., B.J. and R.Z. acknowledge financial support from the Polish National Science Centre (Grant No. 2017/26/M/ ST5/00327). M.A.A. acknowledges financial support from the Polish National Science Centre (Grant No. 2018/30/E/ST5/ 00718). J.M.L. is grateful for financial support from the Spanish MICIN PGC2018-098212-B-C22 and the Catalan DIUE 2017SGR39. The Wroclaw and French teams are indebted to the CNRS for supporting their collaborations in the framework of the ABSOLUTA IEA project. The authors gratefully acknowledge Wroclaw Centre for Networking and Supercomputing and the CCIPL center installed in Nantes (France) for the generous allotment of computer time.

\section{REFERENCES}

(1) Göppert-Mayer, M. Über Elementarakte mit zwei Quantensprungen. Ann. Phys. 1931, 401, 273-294.

(2) Parthenopoulos, D. A.; Rentzepis, P. M. Three-Dimensional Optical Storage Memory. Science 1989, 245, 843-845.
(3) Dvornikov, A. S.; Walker, E. P.; Rentzepis, P. M. Two-Photon Three-Dimensional Optical Storage Memory. J. Phys. Chem. A 2009, $113,13633-13644$.

(4) Denk, W.; Strickler, J.; Webb, W. Two-Photon Laser Scanning Fluorescence Microscopy. Science 1990, 248, 73-76.

(5) Pawlicki, M.; Collins, H. A.; Denning, R. G.; Anderson, H. L. Two-Photon Absorption and the Design of Two-Photon Dyes. Angew. Chem., Int. Ed. 2009, 48, 3244-3266.

(6) Kim, H. M.; Cho, B. R. Small-Molecule Two-Photon Probes for Bioimaging Applications. Chem. Rev. 2015, 115, 5014-5055.

(7) Denk, W.; Strickler, J. H.; Webb, W. W. Two-Photon Laser Scanning Fluorescence Microscopy. Science 1990, 248, 73-76.

(8) Weissleder, R. A Clearer Vision for In Vivo Imaging. Nat. Biotechnol. 2001, 19, 316-317.

(9) Podgorski, K.; Terpetschnig, E.; Klochko, O. P.; Obukhova, O. M.; Haas, K. Ultra-Bright and-Stable Red and Near-Infrared Squaraine Fluorophores for In Vivo Two-Photon Imaging. PLoS One 2012, 7, e51980.

(10) Loudet, A.; Burgess, K. BODIPY Dyes and Their Derivatives: Syntheses and Spectroscopic Properties. Chem. Rev. 2007, 107, 48914932.

(11) Ulrich, G.; Ziessel, R.; Harriman, A. The Chemistry of Fluorescent Bodipy Dyes: Versatility Unsurpassed. Angew. Chem., Int. Ed. 2008, 47, 1184-1201.

(12) Boens, N.; Leen, V.; Dehaen, W. Fluorescent Indicators Based on BODIPY. Chem. Soc. Rev. 2012, 41, 1130-1172.

(13) Frath, D.; Massue, J.; Ulrich, G.; Ziessel, R. Luminescent Materials: Locking $\pi$-Conjugated and Heterocyclic Ligands with Boron(III). Angew. Chem., Int. Ed. 2014, 53, 2290-2310.

(14) Kowada, T.; Maeda, H.; Kikuchi, K. BODIPY-Based Probes for the Fluorescence Imaging of Biomolecules in Living Cells. Chem. Soc. Rev. 2015, 44, 4953-4972.

(15) Ziessel, R.; Ulrich, G.; Harriman, A. The Chemistry of Bodipy: A New El Dorado for Fluorescence Tools. New J. Chem. 2007, 31, 496-501.

(16) Lu, H.; Mack, J.; Yang, Y.; Shen, Z. Structural Modification Strategies for the Rational Design of Red/NIR Region BODIPYs. Chem. Soc. Rev. 2014, 43, 4778-4823.

(17) Boens, N.; Verbelen, B.; Dehaen, W. Postfunctionalization of the BODIPY Core: Synthesis and Spectroscopy. Eur. J. Org. Chem. 2015, 2015, 6577-6595.

(18) Yamaji, M.; Kato, S.-i.; Tomonari, K.; Mamiya, M.; Goto, K.; Okamoto, H.; Nakamura, Y.; Tani, F. Blue Fluorescence from BF2 Complexes of N,O-Benzamide Ligands: Synthesis, Structure, and Photophysical Properties. Inorg. Chem. 2017, 56, 12514-12519.

(19) Cohen, B. J.; Goodman, L. Radiationless Paths in the Diazines. J. Chem. Phys. 1967, 46, 713-721.

(20) Bednarska, J.; Zaleśny, R.; Wielgus, M.; Jędrzejewska, B.; Puttreddy, R.; Rissanen, K.; Bartkowiak, W.; Ågren, H.; Ośmiałowski, B. Two-Photon Absorption of $\mathrm{BF}_{2}$-Carrying Compounds: Insights from Theory and Experiment. Phys. Chem. Chem. Phys. 2017, 19, 5705-5708.

(21) Zaleśny, R.; Szczotka, N.; Grabarz, A.; Ośmiałowski, B.; Jacquemin, D. Design of Two-Photon-Excited Fluorescent Dyes Containing Fluoroborylene Groups. ChemPhotoChem. 2019, 3, 719726.

(22) Horspool, W. M. Photochemistry: Vol. 36; The Royal Society of Chemistry, 2007; Vol. 36, pp 55-90.

(23) Lin, C.-W.; Chou, P.-T.; Liao, Y.-H.; Lin, Y.-C.; Chen, C.-T.; Chen, Y.-C.; Lai, C.-H.; Chen, B.-S.; Liu, Y.-H.; Wang, C.-C.; Ho, M.L. Photoisomerization of a Maleonitrile-Type Salen Schiff Base and Its Application in Fine-Tuning Infinite Coordination Polymers. Chem. - Eur. J. 2010, 16, 3770-3782.

(24) Hamm, P.; Zurek, M.; Röschinger, T.; Patzelt, H.; Oesterhelt, D.; Zinth, W. Femtosecond Spectroscopy of the Photoisomerisation of the Protonated Schiff Base of All-trans Retinal. Chem. Phys. Lett. 1996, 263, 613-621.

(25) Yu, Y.; Yu, C.; Wu, Q.; Wang, H.; Jiao, L.; Wong, W.-Y.; Hao, E. Pure E/Z Isomers of N-Methylpyrrole-Benzohydrazide-Based $\mathrm{BF}_{2}$ 
Complexes: Remarkable Aggregation-, Crystallization-Induced Emission Switching Properties and Application in Sensing Intracellular $\mathrm{pH}$ Microenvironment. J. Mater. Chem. C 2019, 7, 4533-4542.

(26) Bandara, H. M. D.; Burdette, S. C. Photoisomerization in Different Classes of Azobenzene. Chem. Soc. Rev. 2012, 41, 18091825.

(27) Siampiringue, N.; Guyot, G.; Monti, S.; Bortolus, P. The cis $\rightarrow$ trans Photoisomerization of Azobenzene: an Experimental ReExamination. J. Photochem. 1987, 37, 185-188.

(28) Simeth, N. A.; Bellisario, A.; Crespi, S.; Fagnoni, M.; König, B. Substituent Effects on 3-Arylazoindole Photoswitches. J. Org. Chem. 2019, 84, 6565-6575.

(29) Jędrzejewska, B.; Ośmiałowski, B.; Zaleśny, R. Application of Spectroscopic and Theoretical Methods in the Studies of Photoisomerization and Photophysical Properties of the Push-Pull StyrylBenzimidazole Dyes. Photochem. Photobiol. Sci. 2016, 15, 117-128.

(30) Simeth, N. A.; Bellisario, A.; Crespi, S.; Fagnoni, M.; König, B. Substituent Effects on 3-Arylazoindole Photoswitches. J. Org. Chem. 2019, 84, 6565-6575.

(31) Xu, Y.; Gao, C.; Andr'easson, J.; Grøtli, M. Synthesis and Photophysical Characterization of Azoheteroarenes. Org. Lett. 2018, 20, 4875-4879.

(32) Yamaji, M.; Kato, S.-i.; Tomonari, K.; Mamiya, M.; Goto, K.; Okamoto, H.; Nakamura, Y.; Tani, F. Blue Fluorescence from BF2 Complexes of N, O-Benzamide Ligands: Synthesis, Structure, and Photophysical Properties. Inorg. Chem. 2017, 56, 12514-12519.

(33) El-Batta, A.; Jiang, C.; Zhao, W.; Anness, R.; Cooksy, A. L.; Bergdahl, M. Wittig Reactions in Water Media Employing Stabilized Ylides with Aldehydes. Synthesis of $\alpha, \beta$-Unsaturated Esters from Mixing Aldehydes, $\alpha$-Bromoesters, and Ph3P in Aqueous NaHCO3. J. Org. Chem. 2007, 72, 5244-5259.

(34) Grabarz, A. M.; Laurent, A. D.; Jedrzejewska, B.; Zakrzewska, A.; Jacquemin, D.; Ośmiałowski, B. The Influence of the $\pi$ Conjugated Spacer on Photophysical Properties of Difluoroboranyls Derived from Amides Carrying a Donor Group. J. Org. Chem. 2016, $81,2280-2292$.

(35) Chakraborty, A.; Kar, S.; Guchhait, N. Photoinduced Intramolecular Charge Transfer (ICT) Reaction in Trans-Methyl p(Dimethylamino) Cinnamate: A Combined Fluorescence Measurement and Quantum Chemical Calculations. Chem. Phys. 2006, 320, 75-83.

(36) Le Bahers, T.; Adamo, C.; Ciofini, I. A Qualitative Index of Spatial Extent in Charge-Transfer Excitations. J. Chem. Theory Comput. 2011, 7, 2498-2506.

(37) Grabowski, Z. R.; Rotkiewicz, K.; Rettig, W. Structural Changes Accompanying Intramolecular Electron Transfer: Focus on Twisted Intramolecular Charge-Transfer States and Structures. Chem. Rev. 2003, 103, 3899-4032.

(38) Jędrzejewska, B.; Ośmiałowski, B.; Zaleśny, R. Application of Spectroscopic and Theoretical Methods in the Studies of Photoisomerization and Photophysical Properties of the Push-Pull StyrylBenzimidazole Dyes. Photochem. Photobiol. Sci. 2016, 15, 117-128.

(39) Matczyszyn, K.; Olesiak-Banska, J.; Nakatani, K.; Yu, P.; Murugan, N.; Zaleśny, R.; Roztoczyńska, A.; Bednarska, J.; Bartkowiak, W.; Kongsted, J.; Ågren, H.; Samoć, M. One- and Two-Photon Absorption of a Spiropyran - Merocyanine System: Experimental and Theoretical Studies. J. Phys. Chem. B 2015, 119, $1515-1522$.

(40) Alam, M. M.; Chattopadhyaya, M.; Chakrabarti, S. Solvent Induced Channel Interference in the Two-Photon Absorption Process - A Theoretical Study With a Generalized Few-State-Model in Three Dimensions. Phys. Chem. Chem. Phys. 2012, 14, 1156-1165.

(41) Beerepoot, M. T. P.; Alam, M. M.; Bednarska, J.; Bartkowiak, W.; Ruud, K.; Zaleśny, R. Benchmarking the Performance of Exchange-Correlation Functionals for Predicting Two-Photon Absorption Strengths. J. Chem. Theory Comput. 2018, 14, 3677-3685. 